\title{
The BMP inhibitor DAND5 in serum predicts poor survival in breast cancer
}

\author{
Yayun Chi ${ }^{1, *}$, Ling Yao ${ }^{1, *}$, Xin Hu ${ }^{1, *}$, Sheng Huang ${ }^{1}$, Naisi Huang ${ }^{1}$, Shan Li ${ }^{1}$, Zhiming \\ Shao', Jiong Wu ${ }^{2}$ \\ ${ }^{1}$ Department of Breast Surgery, Breast Cancer Institute, Shanghai Cancer Center, Department of Oncology, Shanghai Medical \\ College, Fudan University, Shanghai, 200032, China \\ ${ }^{2}$ Department of Breast Surgery, Breast Cancer Institute, Shanghai Cancer Center, Collaborative Innovation Center of Cancer \\ Medicine, Department of Oncology, Shanghai Medical College, Fudan University, Shanghai 200032, China \\ *These authors have contributed equally to this work \\ Correspondence to: Jiong Wu, e-mail:wujiong1122@vip.sina.com \\ Zhiming Shao e-mail: shaozmyl@126.com
}

Keywords: DAND5, breast cancer, biomarker, secreted factor, prognosis

Received: July 28, $2015 \quad$ Accepted: January 29, $2016 \quad$ Published: February 19, 2016

\section{ABSTRACT}

Background \& Aims: Breast cancer (BC) is prevalent worldwide malignant cancer. Improvements in timely and effective diagnosis and prediction are needed. As reported, secreted DAND5 is contributed to BC metastasis. We aim to assess whether DAND5 in peripheral blood serum could determine BC-specific mortality.

Methods: We used immunohistochemistry staining to detect DAND5 expression in our BC tissue array including 250 samples. Angiogenesis assay and xenograft mice model were used to examine the secreted DAND5 function in BC progression. Serum concentration of DAND5 was examined by ELISA in 1730 BC patients. Kaplan-Meier and adjusted Cox proportional hazards models were utilized to analyze the prognosis and survival of BC patients.

Results: Tissue array results showed that positive DAND5 staining cases displayed a higher likelihood of occurrence of disease events (HR=5.494; 95\% CI: 1.008-2.353; $P=0.048$ ) in univariate analysis and remained the same trend in multivariate analysis ( HR=2.537; 95\% CI: 1.056-6.096; $\mathrm{P}=0.037$ ). DAND5 positive patients exerted generally poor DFS $(P=0.041)$ in the Kaplan-Meier survival analysis. Furthermore, secreted DAND5 promoted tumor growth and angiogenesis in vitro and in vivo. In addition, positive DAND5 in BC patients serum was associated with increased risk of disease events occurrence (univariate: $\mathrm{HR}=1.58$; 95\% CI: 1.206-2.070; $\mathrm{P}=0.001$; multivariate: $\mathrm{HR}=1.4 ; 95 \% \mathrm{CI}: 1.003-1.954 ; \mathrm{P}=0.048$ ) in univariate and multivariate survival analysis. In the Kaplan-Meier analysis, serum DAND5 positively correlated with poor DFS $(P=0.001)$ and DDFS $(P=0.002)$.

Conclusions: DAND5 was correlated with poor survival and could serve as an easily detectable serum biomarker to predict the survival of breast cancer.

\section{INTRODUCTION}

Breast cancer is the most common malignancy among women and represents an important worldwide public health issue $[1,2]$. It is the major cause of cancerrelated death in women and treatment is particularly difficult in patients with tumor metastasis [3]. Despite recent improvements in survival rates, many patients relapse and the majority of these patients die from disseminated metastatic disease [4], [5]. Therefore, there is a disquieting need for the identification of diagnosing markers which can be able to diagnose in early stage of breast carcinogenesis and progression.

Some breast cancer markers are already used in the clinic, such as tissue markers (hormone receptors, human epidermal growth factor-2, urokinase plasminogen activator, plasminogen activator inhibitor, p53 and cathepsin D) and genetic markers (BRAC1 and 2 and gene expression microarray technique, etc.) $[4,6,7]$. Additionally, due to the appropriate price and convenient detection, serum 
markers are widely used in the early screening test. The serum makers (CA 15-3 [8], BR 27-29 [9], MCA, CA 549, carcino embryonic antigen, oncoproteins, and cytokeratins [10]) are used in present diagnosis [11]. Many other secreted factors are used in other tumors, including AFP ( $\alpha$-fetoprotein), CEA (carcinoembryonic antigen) and PSA (prostate-specific antigen). They are all secreted by cancer cells through cellular membranes or exosomes and can be easily detected in the patients' body fluid.

DAND5, a secreted antagonist of TGF- $\beta$ ligands was reported to be critical for $\mathrm{BC}$ metastasis [12]. The aims of the present study were to examine the serum DAND5 concentration in BC patients' peripheral blood and to examine the relationship between DAND5 and $\mathrm{BC}$ patients' prognosis. We tried to find novel serum biomarkers for $\mathrm{BC}$ metastasis and to promptly predict the metastasis and validate their prognosis through conveniently monitoring the biomarkers.

\section{RESULTS}

\section{DAND5 overexpression directly associated with poor disease-free survival}

As reported, antagonist of TGF- $\beta$ ligands DAND5 could induce dormant breast cancer cells to undergo reactivation in the lung. To further validate this result and examine its relationship with breast cancer prognosis, tissue microarrays including $250 \mathrm{BC}$ patients were immunostained for DAND5 (representative images, positive: a-c, negative: $\mathrm{d}$, Figure 1A). Positive staining of DAND5 was detected in $77.2 \%$ ( $\mathrm{n}=193 ; 77.2 \%$ positive, $22.8 \%$ negative) of tumors according to the scoring criterion described in the method. DAND5 mainly localized in cytoplasm and displayed a granular staining in accordance with its role as a secreted protein. We further analyzed the relationship between clinicopathologic features and DAND5 expression level. It showed that DAND5 had a negative relationship with ER status $(\mathrm{P}=0.044)$ and patients age $(\mathrm{P}=0.045)$ (Table 1$)$. There was no correlation between the DAND5 level and menopausal status $(\mathrm{P}=0.052)$, tumor size $(\mathrm{P}=0.769)$, node status $(\mathrm{P}=0.308)$, HER2 status $(\mathrm{P}=0.074)$, differentiation $(\mathrm{P}=0.923)$ and TNM stage $(\mathrm{P}=0.535)$.

To validate the clinical significance of DAND5 overexpression in breast cancer, we analyzed the relationship between DAND5 expression and patient disease-free survival. Both univariate and adjusted multivariate survival analysis revealed statistical significant difference in DAND5 positive and negative group. In this cohort, positive DAND5 staining cases showed a higher likelihood of occurrence of disease events $(\mathrm{HR}=5.494 ; 95 \%$ CI: $1.008-2.353 ; \mathrm{P}=0.048$, Table 2 ) in univariate analysis and remained the same trend in multivariate analysis ( $\mathrm{HR}=2.537$; $95 \%$ CI: $1.056-6.096$; $\mathrm{P}=0.037$, Table 3). Besides, large tumor size also showed a higher likelihood of occurrence of disease events
$(\mathrm{HR}=2.708$; 95\% CI: 1.086-1.715; $\mathrm{P}=0.021$, Table 2) in univariate analysis and remained the same trend in multivariate analysis $(\mathrm{HR}=1.806$; $95 \% \mathrm{CI}$ : $1.085-3.005$; $\mathrm{P}=0.023$, Table 3). Additionally, DAND5 positive patients exerted generally poor DFS $(\mathrm{P}=0.041)$ in the KaplanMeier survival analysis (Figure 1B). The OS showed the same trend although it had no statistical significance $(\mathrm{P}=0.47)$. Furthermore, DAND5 expression was much higher in breast cancer tissues than in normal tissues at both mRNA level and protein level (Supplementary Figure S1). As recent study identified that DAND5 might have important roles in waking dormant breast cancer stem cells in the lung, we further analyzed the data and found that there were 17 patients in 250 tissue array group who finally developed lung metastasis. Only one patient displayed DAND5 negative. All the other 16 patients were turned to be DAND5 positive. These results strongly indicated that DAND5 expression directly associated with recurrent diseases of patients with breast cancer.

\section{DAND5 promoted tumor growth and angiogenesis in vitro and in vivo}

As DAND5 is a secreted BMP inhibitor, we speculated that the secreted DAND5 might affect the capillary network from the surrounding host tissue, which is needed both in cancer proliferation and in cancer metastasis [13]. To test our hypothesis, we constructed the MDA-MB231 stable cell lines with DAND5 overexpression or DAND5 knock down. DAND5 knock down efficiency and over-expression level were confirmed by Western blot in Figure 2C. Using ELISA assay, we first detected the concentration of the secreted DAND5 in cell cultures. The average concentration of MDAMB231 DAND5 overexpression stable cell culture was $12.87 \mathrm{ng} / \mathrm{ml}$, the wild type MDA-MB231 cell line was $2.02 \mathrm{ng} / \mathrm{ml}$ and the DAND5 knocked down cell line was undetectable. HUVEC cells were then treated with these different culture mediums. 48 hours later, angiogenesis was assessed. The data showed HUVECs were organized in a network of pseudocapillary tubes with the DAND5 cell cultures (Figure 2A). DAND5 increased the number of pseudocapillaries in terms of completed circles, while shDAND5 decreased the numbers (Figure 2B for quantification). These data suggest that DAND5 promoted pseudocapillary formation in vitro.

In order to examine whether this function was related with TGF $\beta$ signaling, TGF blocker was used for further examination. However, when TGF $\beta$ blocker Pirfenidone was added, angiogenesis was inhibited. Meanwhile, Pirfenidone inhibited angiogenesis induced by DAND5 (Supplementary Figure S2). As reported, BMPs function was varied in different isoforms and cancers. For example, BMP-2, BMP-4, BMP-6 and BMP-7 are proangiogenic factors, while BMP-9 and BMP-10 are antiangiogenic factors [14]. We speculated that as antagonist 
of TGF $\beta$ ligands and BMP signaling blocker, DAND5 finally induced angiogenensis. The exact mechanism needs further investigation.

To further examine whether DAND5 was related to the cell growth, we carried out the cell proliferation assay with MDA-MB231 cells. The data showed DAND5 knock down MDA-MB231 cells grew slowly compared to the control.
The function of DAND5 was then examined in xenograft mice models. MDA-MB231 scramble cells or MDA-MB231 shDAND5 stable cells were injected into the mice mammary pat to form the xenograft tumors. After 2 weeks, the DAND5 concentration in mice serum in two groups was examined. The average serum concentration of scramble group is $0.29 \mathrm{ng} / \mathrm{ml}$,

\section{DAND5}

A
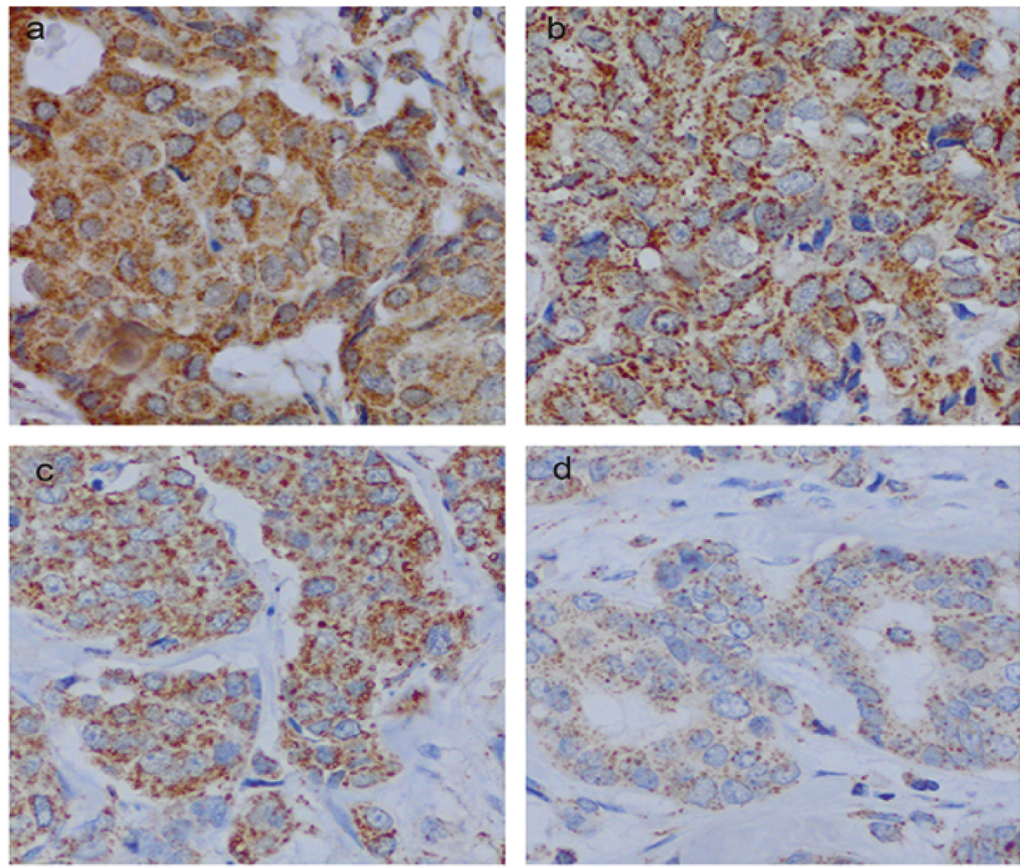

B
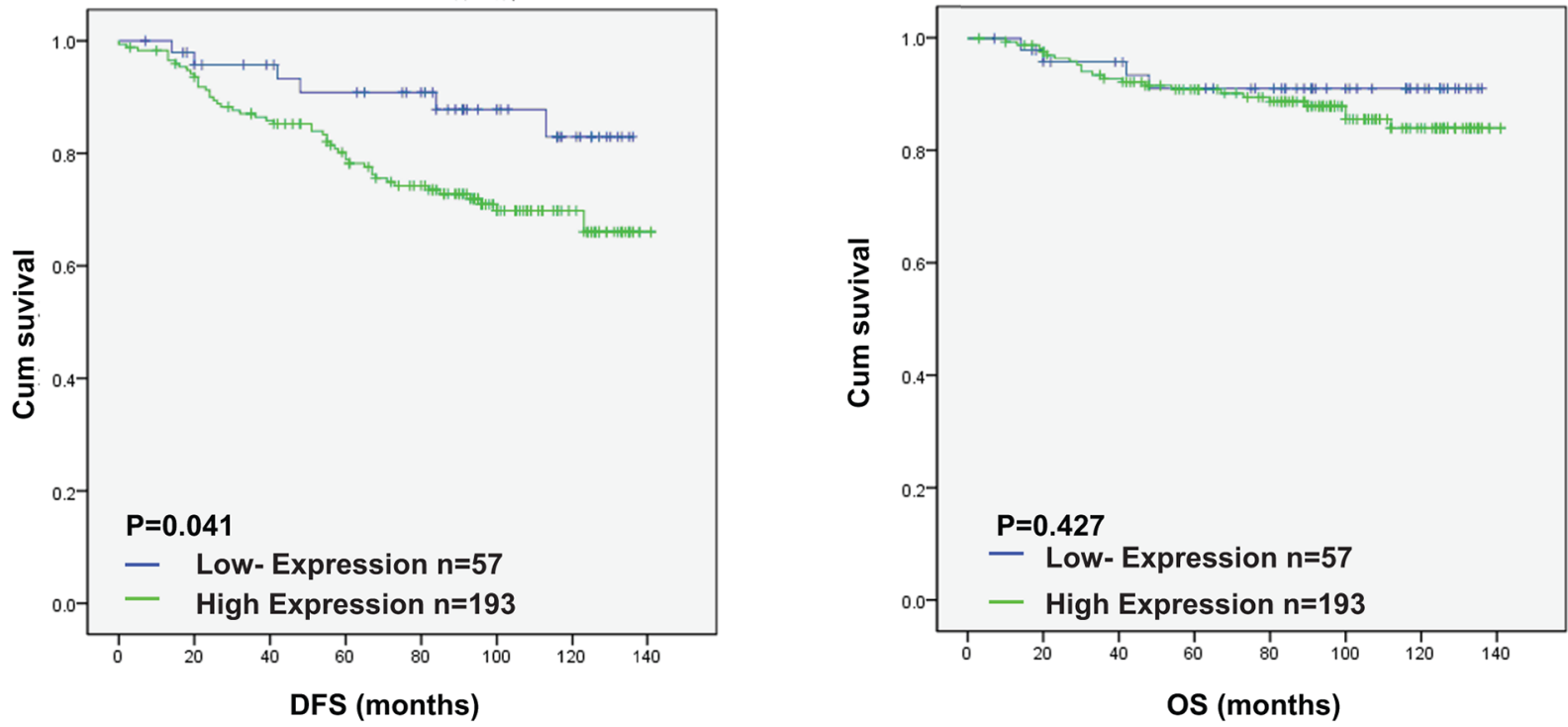

Figure 1: DAND5 expression profile in breast cancer tissue microarrays. A. DAND5 immunostaining was determined in breast cancer and scored as (1) (2) low expression, (3) (4) high expression. All immunohistochemical photomicrographs are magnified $400 \times$. B. Relationship between DAND5 expression and disease free survival (DFS)/overall survival (OS). $P$ values were calculated using the unadjusted log-rank test. 
Table 1: Relationship between DAND5 expression and clinicopathological features in 250 breast cancer patients for IHC detection

\begin{tabular}{|c|c|c|c|c|}
\hline \multirow[t]{2}{*}{ Characteristics } & \multicolumn{2}{|c|}{ DAND5 } & \multirow{2}{*}{$\begin{array}{l}\text { Number of } \\
\text { patients }(\%)\end{array}$} & \multirow[t]{2}{*}{$P^{a}$ value } \\
\hline & Low n (\%) & High n (\%) & & \\
\hline total & $57(22.8)$ & 193(78.2) & 250 & \\
\hline $\begin{array}{l}\text { Age (mean } 51.9, \text { SD } 9.524, \\
\text { median 51, range } 29-85 \text { ) }\end{array}$ & & & & 0.045 \\
\hline$<50$ & $34(13.6)$ & $86(34.4)$ & $120(48)$ & \\
\hline$\geq 50$ & $23(9.2)$ & $107(42.8)$ & $130(52)$ & \\
\hline Menopausal status & & & & 0.052 \\
\hline Pre & $31(12.4)$ & $77(30.8)$ & $108(43.2)$ & \\
\hline Post & $26(10.4)$ & $116(46.4)$ & $142(56.8)$ & \\
\hline Tumor size $(\mathrm{cm})$ & & & & 0.769 \\
\hline$\bigotimes 2 \mathrm{~cm}$ & $25(10)$ & $90(36)$ & $115(46)$ & \\
\hline$>2,5 \bigotimes \mathrm{cm}$ & $28(11.2)$ & $92(36.8)$ & $120(48)$ & \\
\hline$>5 \mathrm{~cm}$ & $4(1.6)$ & $8(3.2)$ & $12(4.8)$ & \\
\hline Node status & & & & 0.308 \\
\hline Negative & $38(15.2)$ & $113(45.2)$ & $151(60.4)$ & \\
\hline Positive & $19(7.6)$ & $78(31.2)$ & $97(38.8)$ & \\
\hline ER status & & & & 0.044 \\
\hline Negative & $40(16)$ & $110(44)$ & $150(60)$ & \\
\hline Positive & $17(6.8)$ & $83(33.2)$ & $100(40)$ & \\
\hline HER-2 status & & & & 0.074 \\
\hline Negative & $34(13.6)$ & $114(45.6)$ & $148(59.2)$ & \\
\hline Positive & $23(9.2)$ & $78(31.2)$ & $100(40)$ & \\
\hline Differentiation & & & & 0.923 \\
\hline I & $1(0.4)$ & $1(0.4)$ & $2(0.8)$ & \\
\hline II & $41(16.4)$ & $143(57.2)$ & $184(73.6)$ & \\
\hline III & $15(6)$ & $46(18.4)$ & $61(24.4)$ & \\
\hline TNM stage & & & & 0.535 \\
\hline I & $17(6.8)$ & $57(22.8)$ & $74(29.6)$ & \\
\hline II & $33(13.2)$ & $99(39.6)$ & $132(52.8)$ & \\
\hline III & $7(2.8)$ & $35(14)$ & $42(16.8)$ & \\
\hline
\end{tabular}

Abbreviations: ER, estrogen receptor; HER-2, human epidermal growth factor receptor 2 a. Based onPearson $\chi 2$ test except for surgery type and radiation therapy, for which P is based on Fisher's exact test.

while shDAND5 groups were undetectable. As shown in Figure 2E and 2F, both tumor size and the MVD (micro-vascular density) were decreased by shDAND5 in the MDA-MB231 shDAND5 group compared to the control groups. DAND5 expression in xenografts was also detected by IHC staining. As shown in Figure $2 \mathrm{G}$, DAND5 expression was significantly decreased in
shDAND5 group compared with control group. Based on these results, we speculated that DAND5 could promote the breast cancer cell growth and induce angiogenesis in breast cancer. The serum DAND5 might be more sensitive and easy to detect and possible to serve as a suitable serum biomarker to predict the prognosis of breast cancer patients. 
Table 2: Univariate regression model of prognostic covariates in $250 \mathrm{BC}$ patients

\begin{tabular}{|c|c|c|c|c|}
\hline \multirow[t]{2}{*}{ Variable } & \multirow[t]{2}{*}{ HR } & \multicolumn{2}{|c|}{$95.0 \% \mathrm{CI}$} & \multirow[t]{2}{*}{$p$ value } \\
\hline & & Lower & Upper & \\
\hline $\operatorname{Age}(<50 />=50)$ & 0.815 & 0.553 & 1.595 & 0.939 \\
\hline Menopausal status(negative/positive) & 0.1 & 0.914 & 2.78 & 1.594 \\
\hline ER(negative/positive) & 0.388 & 0.456 & 1.356 & 0.787 \\
\hline PR(negative/positive) & 0.027 & 0.185 & 0.903 & 0.409 \\
\hline TNM(I, II, III) & 0.003 & 1.24 & 2.767 & 1.852 \\
\hline Pathological stage(I, II, III) & 0.035 & 1.041 & 2.98 & 1.761 \\
\hline Her2 status(negative/positive) & 0.809 & 0.548 & 1.6 & 0.936 \\
\hline Node status(negative/positive) & 0.003 & 1.304 & 3.819 & 2.232 \\
\hline Tumor size $(\bigotimes 2 \mathrm{~cm},>2,5 \bigotimes \mathrm{cm},>5 \mathrm{~cm})$ & 2.708 & 1.086 & 1.715 & 0.021 \\
\hline DAND5 (negative/positive) & 5.494 & 1.008 & 2.353 & 0.048 \\
\hline
\end{tabular}

Abbreviations: CI, confidence interval; ER status, estrogen receptor status; PR status, prostrogen receptor status;HER-2, human epidermal growth factor receptor 2; HR, hazard ratio.

Table 3: Multivariate regression model of prognostic covariates in $250 \mathrm{BC}$ patients

\begin{tabular}{|c|c|c|c|c|}
\hline \multirow[t]{2}{*}{ Variable } & \multirow[t]{2}{*}{ HR } & \multicolumn{2}{|c|}{$95.0 \% \mathrm{CI}$} & \multirow[t]{2}{*}{$p$ value } \\
\hline & & Lower & Upper & \\
\hline $\operatorname{Age}(<50 />=50)$ & 0.573 & 0.284 & 1.155 & 0.12 \\
\hline Menopausal status(negative/positive) & 2.022 & 0.971 & 4.211 & 0.06 \\
\hline ER(negative/positive) & 1.179 & 0.561 & 2.479 & 0.664 \\
\hline PR(negative/positive) & 0.367 & 0.137 & 0.98 & 0.046 \\
\hline TNM(I, II, III) & 1.029 & 0.552 & 1.916 & 0.929 \\
\hline Pathological stage(I, II, III) & 1.594 & 0.887 & 2.865 & 0.119 \\
\hline Her2 status(negative/positive) & 0.874 & 0.481 & 1.586 & 0.657 \\
\hline Node status(negative/positive) & 1.918 & 0.924 & 3.98 & 0.081 \\
\hline Tumor size $(\bigotimes 2 \mathrm{~cm},>2,5 \rrbracket \mathrm{cm},>5 \mathrm{~cm})$ & 1.806 & 1.085 & 3.005 & 0.023 \\
\hline DAND5 (negative/positive) & 2.537 & 1.056 & 6.096 & 0.037 \\
\hline
\end{tabular}

Abbreviations: CI, confidence interval; ER status, estrogen receptor status; PR status, prostrogen receptor status;HER-2, human epidermal growth factor receptor 2; HR, hazard ratio.

\section{Serum DAND5 in breast cancer patients linked to opposing outcomes by ELISA detection}

To test our hypothesis and evaluate the predictive prognostic significance of DNA5 in serum, we performed ELISA detection in peripheral blood (PB) serums of 1730 breast cancer patients who were diagnosed as breast cancer in our institution from 1999 to 2012. As $30.8 \mathrm{pg} / \mathrm{ml}$ was the lowest concentration value of blood sample except zero and undetectable samples, 30.8 $\mathrm{pg} / \mathrm{ml}$ was named as the positive/negative threshold. DAND5 concentration in PB serum higher than 30.8 $\mathrm{pg} / \mathrm{ml}$ was defined as DAND5 positive $(\mathrm{n}=617,35.7 \%)$, the others were DAND5 negative $(n=1113,64.3 \%)$. The clinic characteristics of the patients were shown in Table 4. There was no significant correlation between the expression of DAND5 and the clinicopathological features in the 1730 breast cancer patients which was different from the IHC dataset. We speculated that the trend of secreted DAND5 was not very coordinated with DAND5 expression in breast cancer cell. In univariate and multivariate survival analysis, positive DAND5 expression was associated with a nearly 1.58 -fold and 1.4-fold increased risk of disease events occurrence 
A
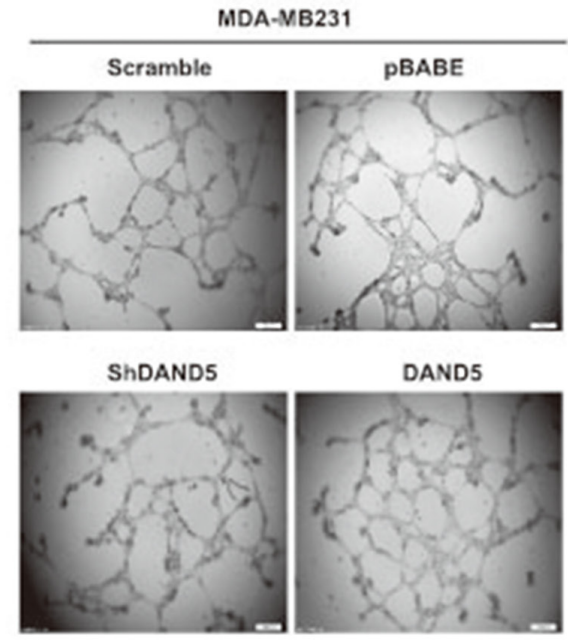

C

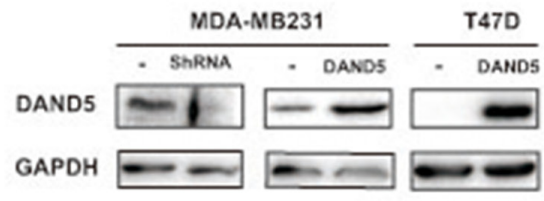

E

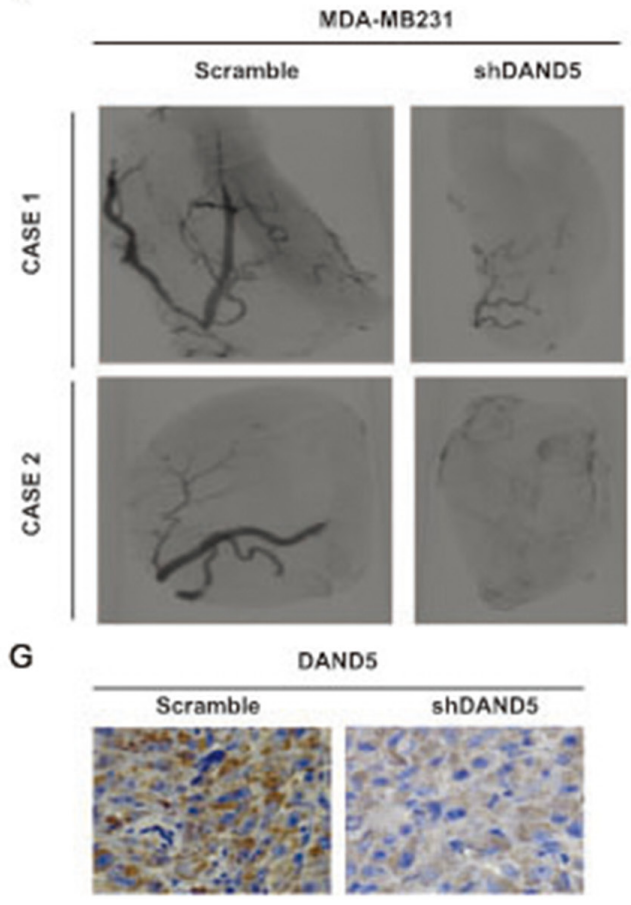

B

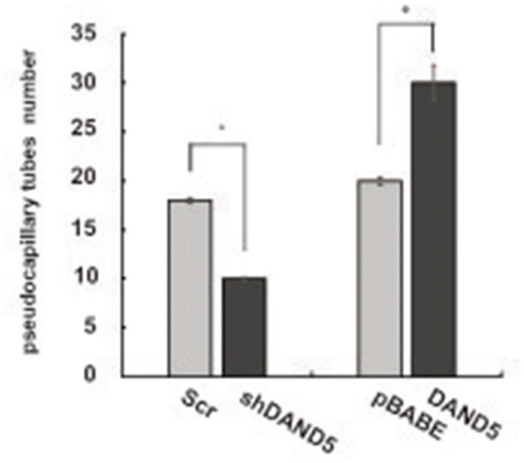

D
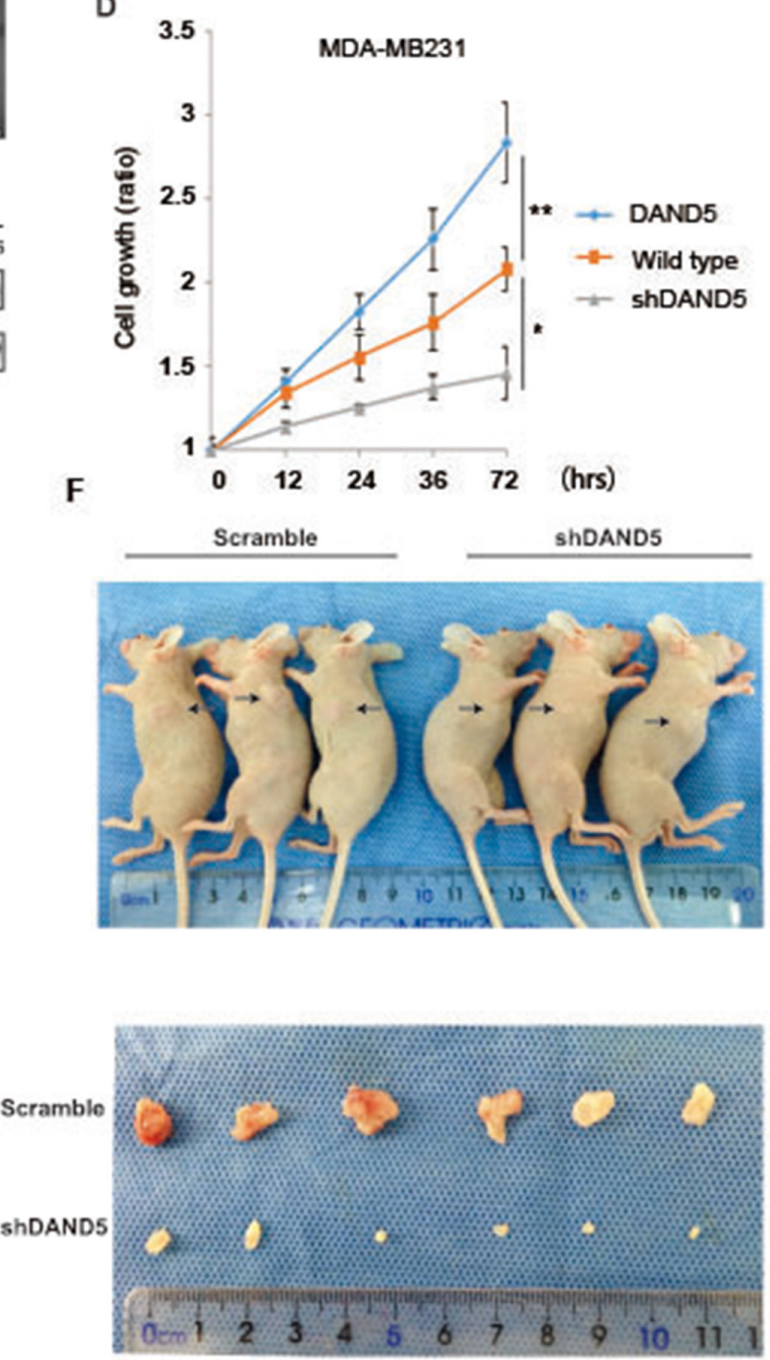

Figure 2: DAND5 promoted tumor proliferation and angiogenesis in vitro and in vivo. A. Representative pictures of pseudocapillary formation in matrigel from HUVECs in $0.1 \%$ FBS exposed to breast cancer cell culture at $12 \mathrm{~h}$ after cell seeding. B. Quantification of pseudocapillaries obtained by counting numbers of complete circles/wells. Numbers represent the mean of 6 samples \pm SEM of three experiments run in triplicate. C. MDA-MB231 DAND5 and knock down stable cell lysates and T47D DAND5 stable cell lysates were immunoblotted using DAND5 antibody. D. MDA-MB231 wild type, DAND5 and knock down stable cells were seeded in 96 well plate and cultured for the times as indicated, the cell growth was analyzed with $\mathrm{CCK}-8$ assay. ${ }^{*} \mathrm{P}<0.05$. ${ }^{* *} \mathrm{P}<0.001$. E. Inhibition of DAND5 by shRNA inhibited the vascularization of tumors in mice. The images were reconstructed using the filtered back projection (FBP) algorithm. Tumorigenesis was obtained after injection of MDA-MB231 cells or MDA-MB231 shDAND5 cells. F. Image of representative tumors from MDA-MB231 or MDA-MB231 shDAND5 xenografts harvested at end point. G. Images to visualize positive staining of DAND5 in xenograft tumor harvested at end point. Bars: 20 um, magnification $\times 400$. 
Table 4: Relationship between DAND5 expression in peripheral blood serums and clinicopathological features in 1730 breast cancer patients for ELISA detection

\begin{tabular}{|c|c|c|c|c|}
\hline \multirow[t]{2}{*}{ Characteristics } & \multicolumn{2}{|c|}{ DNAD5 } & \multirow{2}{*}{$\begin{array}{c}\text { Number of } \\
\text { patients }(\%)\end{array}$} & \multirow[t]{2}{*}{ P value } \\
\hline & Low n (\%) & High n (\%) & & \\
\hline Total & $1113(64.3)$ & $617(35.7)$ & 1730 & \\
\hline Age & & & & 0.583 \\
\hline$<50$ & $1011(58.4)$ & $517(29.9)$ & $158(88.3)$ & \\
\hline$\geq 50$ & $93(5.4)$ & $47(2.7)$ & $140(8.1)$ & \\
\hline Menopausal status & & & & 0.191 \\
\hline Pre & $542(31.3)$ & $283(16.4)$ & $825(47.6)$ & \\
\hline Post & $562(32.5)$ & $335(19.4)$ & 897(1.9) & \\
\hline Tumor size $(\mathrm{cm})$ & & & & 0.305 \\
\hline$\bigotimes 2 \mathrm{~cm}$ & $559(32.3)$ & $283(16.4)$ & $842(48.7)$ & \\
\hline$>2,5 \bigotimes \mathrm{cm}$ & $369(21.3)$ & $235(14.6)$ & $604(35.9)$ & \\
\hline$>5 \mathrm{~cm}$ & $32(1.8)$ & $20(1.2)$ & $52(3)$ & \\
\hline Node status & & & & 0.196 \\
\hline Negative & $698(40.3)$ & $362(20.9)$ & $1060(61.2)$ & \\
\hline Positive & $407(23.5)$ & $256(14.8)$ & $663(38.3)$ & \\
\hline ER status & & & & 0.463 \\
\hline Negative & $320(18.5)$ & $168(9.7)$ & $488(28.2)$ & \\
\hline Positive & $718(41.5)$ & $412(23.8)$ & $1130(65.3)$ & \\
\hline HER-2 status & & & & 0.164 \\
\hline Negative & $834(48.2)$ & $487(28.2)$ & $1321(76.4)$ & \\
\hline Positive & $71(4.1)$ & $30(1.7)$ & $101(5.8)$ & \\
\hline Differentiation & & & & 0.130 \\
\hline 凶l & $20(1.2)$ & $8(0.5)$ & $28(1.7)$ & \\
\hline II & $500(28.9)$ & $305(17.6)$ & $805(46.5)$ & \\
\hline III & $280(16.2)$ & $135(7.8)$ & $415(24)$ & \\
\hline TNM stage & & & & 0.091 \\
\hline I & $390(22.5)$ & $184(10.6)$ & $574(33.1)$ & \\
\hline II & $407(23.5)$ & $239(13.8)$ & $646(47.3)$ & \\
\hline III & $174(10.1)$ & $117(6.8)$ & 291(16.9) & \\
\hline
\end{tabular}

Abbreviations: ER, estrogen receptor; HER-2, human epidermal growth factor receptor 2 a. Based onPearson $\chi 2$ test except for surgery type and radiation therapy, for which P is based on Fisher's exact test.

(univariate: $\mathrm{HR}=1.58 ; 95 \% \mathrm{CI}: 1.206-2.070 ; \mathrm{P}=0.001$; multivariate: $\mathrm{HR}=1.4 ; 95 \% \mathrm{CI}: 1.003-1.954 ; \mathrm{P}=0.048$, Table 5). Furthermore, in the 1730 patients group, there were 63 patients who developed lung metastasis. Only 3 patients displayed secreted DAND5 negative. All the other 60 patients were turned to be secreted DAND5 positive. In the Kaplan-Meier analysis, except OS $(\mathrm{P}=0.523$; Figure $3 \mathrm{C})$, DAND5 positively correlated with poor DFS ( $\mathrm{P}=0.001$; Figure $3 \mathrm{~A})$ and DDFS $(\mathrm{P}=0.002$; Figure $3 \mathrm{~B}$ ), revealing a possibility that DAND5 expression was linked to poor prognosis in $\mathrm{BC}$.

\section{DISCUSSION}

DAND5, directly binding to BMP proteins functions as an antagonist of BMP. It has been reported that DAND5 
Table 5: Univariate and multivariate analysis for disease free survival in $\mathbf{1 7 3 0}$ cases

\begin{tabular}{|c|c|c|c|c|}
\hline & \multicolumn{2}{|c|}{ Univariate analysis } & \multicolumn{2}{|c|}{ Multivariate analysis } \\
\hline & HR $(95 \%$ CI $)$ & p value & HR (95\% CI) & p value \\
\hline Age & $0.661(0.206-2.117)$ & 0.485 & $1.106(0.593-2.062)$ & 0.751 \\
\hline Menopausal status & $0.996(0.592-1.676)$ & 0.989 & $0.800(0.568-1.128)$ & 0.203 \\
\hline Tumor grade & $4.286(2.313-7.942)$ & $<0.001$ & $4.462(2.097-10.278)$ & $<0.001$ \\
\hline Tumor size & $3.317(2.242-4.907)$ & $<0.001$ & $1.613(1.159-2.245)$ & 0.004 \\
\hline Lymph node status & $2.847(2.276-3.563)$ & $<0.001$ & $2.135(1.552-2.938)$ & $<0.001$ \\
\hline ER status & $0.399(0.236-0.674)$ & 0.001 & $0.861(0.596-1.242)$ & 0.423 \\
\hline HER-2/neu status & $2.304(0.905-5.867)$ & 0.08 & $1.122(0.584-2.153)$ & 0.73 \\
\hline DAND5 & $1.58(1.206-2.070)$ & 0.001 & $1.4(1.003-1.954)$ & 0.048 \\
\hline
\end{tabular}

Abbreviations: HR, hazard ratio; CI, confidence interval; ER, estrogen receptor; HER-2, human epidermal growth factor receptor 2; a.p is based on the Cox regression test.

A

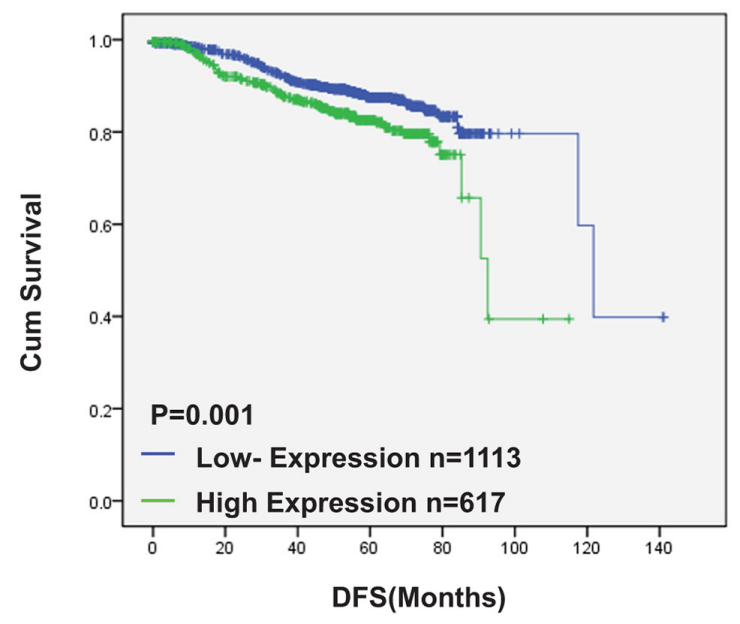

B

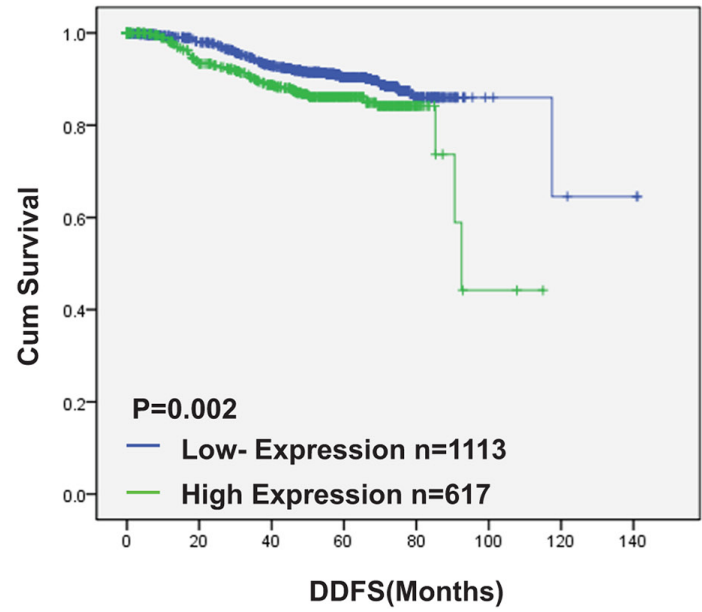

C

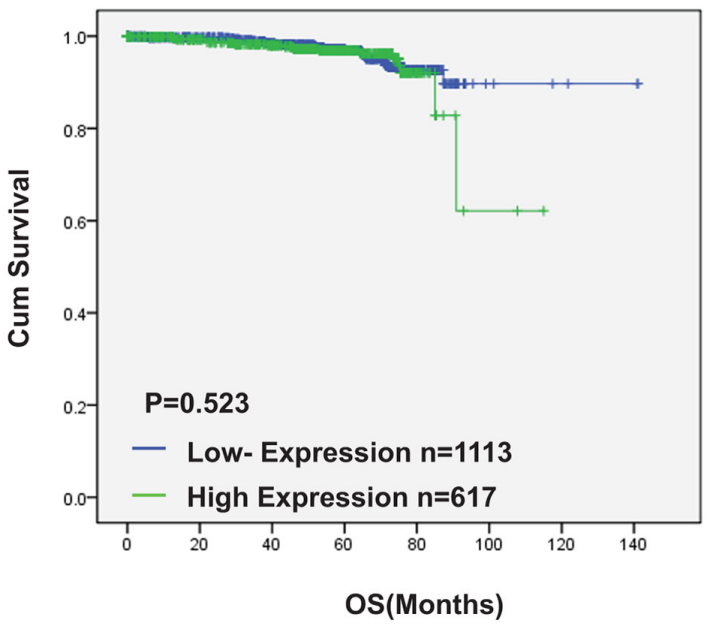

Figure 3: Serum DAND5 in breast cancer patients linked to opposing outcomes by ELISA detection. Relationship between DAND5 expression and disease free survival (DFS)/overall survival (OS). $P$ values were calculated using the unadjusted log-rank test. 
is involved in regulating organogenesis, body patterning, and tissue differentiation [15-17]. One recent report revealed that DAND5 was involved in the metastasis in breast cancer [18]. In the present study, we found that the prognosis of breast cancer patients was closely related with DAND5 expression not only in the cancer tissues but also in the peripheral blood (PB) serums. DAND5 could serve as a predict serum maker in breast cancer.

As we know, many steps are critical for the formation of metastasis. Growth of neoplastic cells must be progressive, with nutrients for the expanding tumor mass initially supplied by simple diffusion. Extensive vascularization must occur and the synthesis and secretion of angiogenic factors should establish a capillary network from the surrounding host tissue. The cells can then invade blood vessels, enter the circulation and produce additional metastasis $[13,19,20]$. Therefore, the microenvironment is very important for the $\mathrm{BC}$ progression [21-23]. One of the factors which could influence the microenvironment is the secreted factor family [24, 25]. Cancer secreted factors can change the cancer microenvironment to promote metastasis $[26,27]$. Therefore, secreted factors which were related to the recurrence and progression of diseases could be helpful biomarkers for the diagnosis and prediction.

Till now, many new secreted factors have been discovered and used for cancer predictions. A panel of miRNAs combined with CA19-9 is established to be serum biomarker in pancreatic cancer [28]. Serum Enterolactone is reported to be related with the prognosis of postmenopausal breast cancer [29]. Tumor-induced miRNA16 and miRNA378 secretion changes as regulators and biomarkers of osteolytic bone metastasis [30]. Besides, serum DKK1 is found to serve as a novel protein biomarker for the diagnosis of hepatocellular carcinoma [31].

Our group is always interested in identifying novel serum biomarkers of breast cancer. When Gao and colleagues found that DAND5, a secreted factor induced dormant breast cancer cells to undergo reactivation in the lung through a gain-of-function cDNA screen, we was highly inspired. We first validated the DAND5 function using our institute tissue array including 250 breast cancer patients. It showed that DAND5 expression in the cancer tissue directly associated with recurrent diseases of $\mathrm{BC}$ patients.

We then examined the function of secreted DAND5 in the microenvironment. When DAND5 was stably expressed in the breast cancer cells, the concentration of DAND5 in the culture medium of these cells significantly increased. The secreted DAND5 increased the number of pseudocapillaries of HUVEC cells in terms of completed circles, while shDAND5 decreased the numbers. It suggested that secreted DAND5 could promote pseudocapillary formation. In the xenograft mice model, DAND5 overexpression also promoted the microvascular formation in the xenograft tumors. Additionally, the tumors in the DAND5 group were all bigger than the control group. These results suggest that the secreted DAND5 regulated the cancer progression through changing the microenvironment.

To test our hypothesis, the validation model which contained 1730 breast cancer patients' peripheral blood (PB) serums was established. The serum concentration of DAND5 was detected through ELISA assay. In healthy control group, DAND5 concentration was extremely low and undetectable. In univariate and multivariate survival analysis, positive DAND5 expression was associated with a nearly 1.58 -fold and 1.4-fold increased risk of disease events occurrence. Using the Kaplan-Meier analysis, we found that DAND5 positivity correlated with poor DFS and DDFS in breast cancer.

Taken together, we speculate that when DAND5 gene was amplified or DAND5 protein was overexpressed in breast cancer, DAND5 could be secreted from the cells into the surrounding and then affect the microenvironment to facility the cancer cell for progression. DAND5 could serve as an easily detectable serum biomarker for early prognosis and to predict the survival of breast cancer patients.

\section{MATERIALS AND METHODS}

\section{Patients}

Informed consent forms were signed by each participant, and appropriate ethical committee approval was obtained. For the tissue microarray, 250 primary BC tissue samples from female invasive ductal carcinoma patients (no co-morbidities reported) were randomly collected at the Department of Breast Surgery of the Fudan University Shanghai Cancer Center (FDUSCC, Shanghai, P.R. China) between 2002 and 2006. The median follow-up time was 96 months (84-141 months). All patients were female and had a median age of 53 years at the time of diagnosis. Prior to constructing the tissue microarray (TMA), each paraffin-embedded tumor sample was defined and the tumor regions marked based on H\&E staining. The TMA sections were generated by the Department of Pathology at the Fudan University Shanghai Cancer Center. Briefly, tissue cylinders with diameters of $10 \mathrm{~mm}$ were punched from the above regions and transferred to recipient array blocks using a Tissue Micro Arrayer. The TMA included duplicate cores from different areas of the same tumor to compare the staining patterns.

For the DAND5 serum ELISA assay, 1730 blood samples were randomly collected from BC patients before primary breast surgery at the Department of Breast Surgery in FDUSCC between 1999 and 2012. The median follow-up time was 54.9 months (13-159 months). All patients were female and had a median age of 51 years at the time of diagnosis. Serum samples were aliquoted and 
stored at $-80^{\circ} \mathrm{C}$ until DAND5 was analyzed via sandwich ELISA as described below.

\section{Immunohistochemistry}

Expression levels of DAND5 (TA503555, ORIGENE) in postoperative paraffin-embedded tumor specimens from breast cancer patients and mice tumor tissues were detected with IHC. The concentrations of antibodies used are as follows: DAND5, 1:100. The Envision and diaminobenzidine (DAB) Color Kit was purchased from Gene Tech Company Limited (Shanghai, China). The staining procedures strictly followed the supplier's recommendation. The staining index (SI, range $0-9)$ was considered as the product of the intensity score ( 0 , no staining; $1+$, faint/equivocal; $2+$, moderate; $3+$, strong) and the distribution score (0, no staining; $1+$, staining of $<10 \%$ of cells; $2+$, between $10 \%$ and $50 \%$ of cells; and $3+,>50 \%$ of cells). For DAND5 protein in this study, a moderate/strong staining $(\mathrm{SI}=3-9)$ was defined as positive or high staining, and a weak or negative staining $(\mathrm{SI}=0-2)$ was defined as negative or low staining.

\section{ELISA}

A Human DAND5 Duo Set kit for ELISA (R\&D Systems, Inc., USA) was used to perform serum DAND5 ELISA assays according to the manufacturer's protocol. Briefly, the plates were coated with the DAND5 capture antibody in PBS. After incubation overnight at $4{ }^{\circ} \mathrm{C}$, the plates were then washed and blocked with washing buffer. Subsequently, $10 \mu \mathrm{l}$ of standard preparations of different densities, as well as patient blood serum samples, were added to the wells, and the plate was incubated overnight at $4^{\circ} \mathrm{C}$. After the plate was washed, a $100-\mu 1$ solution containing the detection antibody $(300 \mu \mathrm{g} / \mathrm{ml})$ was added and incubated at room temperature for $2 \mathrm{hrs}$. The wells were washed again and then incubated with a $100-\mu 1$ solution containing streptavidin-conjugated horseradish peroxidase at room temperature for $20 \mathrm{~min}$. Following multiple washes, the wells were treated with $100 \mu \mathrm{l}$ of substrate solution and incubated in the dark at room temperature for $20 \mathrm{~min}$. After the addition of $50 \mu \mathrm{l}$ of stop solution to each well, the absorbance was detected at 450 nm. The online tool "Four Parameter Logistic Fit" was used to calculate the absolute concentration of DAND5 in the blood serum samples.

\section{In vitro angiogenesis model}

Human Umbilical Vein Endothelial Cells (HUVEC) were suspended in culture medium from stable cell lines and then plated onto a thin layer (300 $\mathrm{ml}$ ) of basement membrane matrix (Matrigel; BD Biosciences) in 24-well plates at $1 \times 10^{4}$ cells/well. After $12 \mathrm{~h}$, the medium was removed, cells were fixed, and images of cells were obtained with a light microscope
(Leica) at $\times 20$ magnification. Quantification of the tubular structures was performed by counting the number of complete circles produced by interlinking tubular HUVECs [15].

\section{Tumorigenicity assays and blood vessel assessment in athymic mice}

Female athymic BALB/c nu/nu mice, 4-6 weeks old, were obtained from the Shanghai Institute of Materia Medica, Chinese Academy of Sciences. All studies on mice were conducted in accordance with the National Institute of Health (NIH) 'uide for the Care and Use of Laboratory Animals'. The study protocol was approved by the Shanghai Medical Experimental Animal Care Committee. Animals were divided into four groups: MDA-MB-231/pBABE and MDA-MB-231/DAND5, MDA-MB-231/scramble and MDA-MB-231/shDAND5. Each group contained 16 mice. Cells (MDA-MB-231, $1.5 \times 10^{6}$ ) were injected into the mammary fat pad of mice. Animals were monitored every 2 days for tumor growth and general health. Animals were sacrificed and autopsied at 6 weeks after cell inoculation. To confirm the expression of the indicated proteins, sections were cut at $50 \mu \mathrm{m}$ intervals and stained with hematoxylin and eosin (H\&E) and by IHC. Microangiography for blood vessels was performed at the Beamline BL13W1, and $\mathrm{X}$-ray imaging at the biomedical application station of the Shanghai Synchrotron Radiation Facility (SSRF) in China. The maximum light size of the beam was $45 \mathrm{~mm}$ (horizontal) $\times 5 \mathrm{~mm}$ (vertical) at the object position at $16 \mathrm{keV}$. All animals were anesthetized by intraperitoneal injection of ketamine $(200 \mathrm{mg} / \mathrm{kg}$ ) (Ketanest; Pfizer, Karlsruhe, Germany). The image contrast agent microfil (Flow Tech Inc., Carver, USA) was injected into the left ventricle. Serial images of tumor blood vessels in nude mice were then recorded using SSRF.

\section{Statistical analyses}

Correlations between clinical-pathological parameters and interested markers were evaluated using contingency tables and Pearson $\chi 2$ test or Fisher's exact test. Disease-free survival and overall survival were derived from the Kaplan-Meier estimate and compared by the log-rank test. Univariate and multivariable analysis were carried out using the Cox risk proportion model. Statistics was analyzed using SPSS (version 13.0; SPSS Company). All P values are two-sided and a P value of less than 0.05 was considered significant.

\section{ACKNOWLEDGMENTS}

We thank Dr. Filippo G. Giancotti for the Coco and shCoco plasmids. This work was supported by 
the National Natural Scientific Foundation of China (81102002) and the National Basic Research Program of China (2010CB834305, 2010CB834301).

\section{CONFLICTS OF INTEREST} interests.

The authors declare that they have no competing

\section{GRANT SUPPORT}

This study was supported by Yayun Chi's National Natural Scientific Foundation of China (81102002) and Jiong Wu's National Basic Research Program of China (2010CB834305, 2010CB834301).

\section{REFERENCES}

1. Torre LA, Bray F, Siegel RL, Ferlay J, Lortet-Tieulent J, Jemal A. Global cancer statistics. CA Cancer J Clin. 2015; 65:87-108

2. Siegel RL, Miller KD, Jemal A. Cancer statistics, 2015. CA Cancer J Clin. 2015; 65:5-29.

3. Johnson CJ, Graff R, Moran P, Cariou C, Bordeaux $\mathrm{S}$. Breast cancer stage, surgery, and survival statistics for Idaho's National Breast and Cervical Cancer Early Detection Program population, 2004-2012. Prev Chronic Dis. 2015; 12:E36.

4. Dubey AK, Gupta U, Jain S. Breast cancer statistics and prediction methodology: a systematic review and analysis. Asian Pac J Cancer Prev. 2015; 16:4237-45.

5. Shim HJ, Kim SH, Kang BJ, Choi BG, Kim HS, Cha ES, Song BJ. Breast cancer recurrence according to molecular subtype. Asian Pac J Cancer Prev. 2014; 15:5539-44.

6. McCullough AE, Dell'Orto P, Reinholz MM, Gelber RD, Dueck AC, Russo L, Jenkins RB, Andrighetto S, Chen B, Jackisch C, Untch M, Perez EA, Piccart-Gebhart MJ, Viale G. Central pathology laboratory review of HER2 and ER in early breast cancer: an ALTTO trial [BIG 2-06/NCCTG N063D (Alliance)] ring study. Breast Cancer Res Treat. 2014; 143:485-92.

7. Huang L, Liu Z, Chen S, Liu Y, Shao Z. A prognostic model for triple-negative breast cancer patients based on node status, cathepsin-D and Ki-67 index. PLoS One. 2013; 8:e83081.

8. Fejzic H, Mujagic S, Azabagic S, Burina M. Tumor marker CA 15-3 in breast cancer patients. Acta Med Acad. 2015; 44:39-46.

9. Klee GG, Schreiber WE. MUC1 gene-derived glycoprotein assays for monitoring breast cancer (CA 15-3, CA 27.29, BR): are they measuring the same antigen? Arch Pathol Lab Med. 2004; 128:1131-5.

10. Gudlaugsson E, Klos J, Skaland I, Janssen EA, Smaaland R, Feng W, Shao Z, Malpica A, Baak JP. Prognostic comparison of the proliferation markers (mitotic activity index, phosphohistone H3, Ki67), steroid receptors, HER2, high molecular weight cytokeratins and classical prognostic factors in $\mathrm{T}(1)(-)(2) \mathrm{N}(0) \mathrm{M}(0)$ breast cancer. Pol J Pathol. 2013; 64:1-8.

11. Bao H, Yu D, Wang J, Qiu T, Yang J, Wang L. Predictive value of serum anti-p53 antibodies, carcino-embryonic antigen, carbohydrate antigen 15-3, estrogen receptor, progesterone receptor and human epidermal growth factor receptor-2 in taxane-based and anthracycline-based neoadjuvant chemotherapy in locally advanced breast cancer patients. Anticancer Drugs. 2008; 19:317-23.

12. Martinez J, Zhang XH. BMP/Coco antagonism as a deterministic factor of metastasis dormancy in lung. Breast Cancer Res 2013;15:302.

13. Fidler IJ. The pathogenesis of cancer metastasis: the 'seed and soil' hypothesis revisited. Nat Rev Cancer. 2003; 3:453-8.

14. Beets K, Huylebroeck D, Moya IM, Umans L, Zwijsen A. Robustness in angiogenesis: notch and BMP shaping waves. Trends Genet. 2013; 29:140-9.

15. Seton-Rogers S. Metastasis: Recharging with COCO. Nat Rev Cancer. 2012; 12:655.

16. Bates TJ, Vonica A, Heasman J, Brivanlou AH, Bell E. Coco regulates dorsoventral specification of germ layers via inhibition of TGFbeta signalling. Development. 2013; 140:4177-81.

17. Deglincerti A, Haremaki T, Warmflash A, Sorre B, Brivanlou AH. Coco is a dual-activity modulator of TGFbeta signaling. Development. 2015; 142:2678-85. doi: 10.1242/dev.122358.

18. Gao H, Chakraborty G, Lee-Lim AP, Mo Q, Decker M, Vonica A, Shen R, Brogi E, Brivanlou AH, Giancotti FG. The BMP inhibitor Coco reactivates breast cancer cells at lung metastatic sites. Cell. 2012; 150:764-79.

19. Hanahan D, Weinberg RA. Hallmarks of cancer: the next generation. Cell. 2011; 144:646-74.

20. Hainaut P, Plymoth A. Targeting the hallmarks of cancer: towards a rational approach to next-generation cancer therapy. Curr Opin Oncol. 2013; 25:50-1.

21. Linton SS, Sherwood SG, Drews KC, Kester M. Targeting cancer cells in the tumor microenvironment: opportunities and challenges in combinatorial nanomedicine. Wiley Interdiscip Rev Nanomed Nanobiotechnol. 2015.

22. Romaniuk A, Lsmall U CIUM. Immune microenvironment as a factor of breast cancer progression. Diagn Pathol. 2015; 10:79.

23. Clark AG, Vignjevic DM. Modes of cancer cell invasion and the role of the microenvironment. Curr Opin Cell Biol. 2015; 36:13-22.

24. Roma-Rodrigues C, Fernandes AR, Baptista PV. Exosome in tumour microenvironment: overview of the crosstalk between normal and cancer cells. Biomed Res Int. 2014; 2014:179486. 
25. Singh R, Pochampally R, Watabe K, Lu Z, Mo YY. Exosome-mediated transfer of miR-10b promotes cell invasion in breast cancer. Mol Cancer. 2014; 13:256.

26. Banerjee S, Sengupta K, Dhar K, Mehta S, D'Amore PA, Dhar G, Banerjee SK. Breast cancer cells secreted platelet-derived growth factor-induced motility of vascular smooth muscle cells is mediated through neuropilin-1. Mol Carcinog. 2006; 45:871-80.

27. Murakami N, Koufuji K, Shirouzu K. Influence of hepatocyte growth factor secreted from fibroblasts on the growth and invasion of scirrhous gastric cancer. Int Surg. 2001; 86:151-7.

28. Schultz NA, Dehlendorff C, Jensen BV, Bjerregaard JK, Nielsen KR, Bojesen SE, Calatayud D, Nielsen SE, Yilmaz M, Hollander NH, Andersen KK, Johansen JS. MicroRNA biomarkers in whole blood for detection of pancreatic cancer. JAMA. 2014; 311:392-404.

29. Buck K, Vrieling A, Zaineddin AK, Becker S, Husing A, Kaaks R, Linseisen J, Flesch-Janys D, Chang-Claude J. Serum enterolactone and prognosis of postmenopausal breast cancer. J Clin Oncol. 2011; 29:3730-8.

30. Ell B, Mercatali L, Ibrahim T, Campbell N, Schwarzenbach H, Pantel K, Amadori D, Kang Y. Tumor-induced osteoclast miRNA changes as regulators and biomarkers of osteolytic bone metastasis. Cancer Cell. 2013; 24:542-56.

31. Shen Q, Fan J, Yang XR, Tan Y, Zhao W, Xu Y, Wang N, Niu Y, Wu Z, Zhou J, Qiu SJ, Shi YH, Yu B, and et al. Serum DKK1 as a protein biomarker for the diagnosis of hepatocellular carcinoma: a large-scale, multicentre study. Lancet Oncol. 2012; 13:817-26. 